\title{
Lévy simple structural models
}

\section{Martin Baxter ${ }^{1}$}

\section{Nomura Fixed Income Quant Group 22 December 2006}

\section{Introduction}

The Gaussian copula model does not fit the credit market, and the base correlation method of forcing it to fit has several disadvantages. Its main drawbacks are a theoretical possibility of arbitrage; actual arbitrage in practice; and difficulties in extending the method to price bespoke baskets and more exotic products.

In this paper we will propose a family of Levy structural models which fit the market better than the Gaussian copula. These models are intuitive, easy to implement, and provide insights into both risk management and relative value opportunities. They also extend to bespoke baskets and more exotic products.

The economic idea behind the model is that the tails of the Gaussian distribution are too thin to model the credit market accurately. Although the Gaussian distribution is widely used in other asset classes, it is rarely suitable for extreme out-of-the-money options. But almost all credit default events are extreme events which are controlled by the tail of the distribution. For this reason we reject Brownian motion and look for alternative stochastic processes which have heavier tails.

As movements in the credit market are sometimes sudden and jump-like, we choose to work with Levy processes. These can have both jumps and heavier tails, as we desire. We will also show a way of creating multi-variate Levy processes, which will allow us to break up an entity's jumps into global jumps and idiosyncratic jumps. This mirrors the reality of market movements - May 2005 was a global jump and Parmalat was an idiosyncratic jump.

There has been growing interest recently in studying jump models of individual names. Joshi and Stacey (2005) developed a method with a global time-scale factor (intensity gamma) and they achieve CDO calibration. An economic drawback is that it does not allow idiosyncratic spread jumps and its practical limitation is that it requires Monte Carlo. Schoutens (Jan 2006) drives the intensity as a gamma OU process with spread up-jumps, and has success with CDS calibration, but not CDO calibration. Luciano and Schoutens (Dec 2005) have a multivariate gamma process also using a global time-change clock. This also has no idiosyncratic spread jumps, and does not match CDO prices.

\section{Levy processes}

Let us give a brief summary of the basic properties of a Levy process. Winkel (2004) gives a brief introduction, and Applebaum (2004) is a very useful reference for Levy processes. Levy processes are, in some sense, a generalisation of Brownian motion to contain drift, Brownian motion, and jump terms. Formally a stochastic process $X(t)$ is a Levy process if

- $X(0)=0$,

- $X$ has stationary increments, that is $X(s+t)-X(s) \stackrel{d}{=} X(t)$,

\footnotetext{
${ }^{1}$ Nomura International plc, 1 St Martin's-le-Grand, London EC1A 4NP.

Email:work@martinbaxter.co.uk
} 
- $\quad X$ has independent increments, that is $X(s+t)-X(s)$ is independent of $(X(u): u \leq s)$, and

- (technical continuity condition), $\lim _{t \rightarrow s} P\left(\left|X_{t}-X_{s}\right|>\varepsilon\right)=0$, for all positive $\varepsilon$.

Given sufficient boundedness on $X$, its moment generating function can be written as

$$
E\left(\exp \left(\theta X_{t}\right)\right)=\exp (t \psi(\theta))
$$

where the Levy symbol of $X$ is $\psi(\theta)$ which can be decomposed as

$$
\psi(\theta)=\mu \theta+\frac{1}{2} \sigma^{2} \theta^{2}+\int_{-\infty}^{\infty}(\exp (\theta x)-1) v(x) d x
$$

Its three terms correspond to:

- Constant drift, where $\mu$ is the drift coefficient

- Brownian motion, where $\sigma$ is the volatility

- Pure-jump term, where the Levy measure $v$ gives the intensity of the Poisson arrival process of jumps of size $x$. That is, jumps whose size lies in $[x, x+d x]$ occur as a Poisson arrival process with intensity $v(x) d x$.

A useful basic Levy process is the Gamma process, which is a pure-jump increasing process with Levy measure

$$
\begin{gathered}
v(x)=\gamma x^{-1} \exp (-\lambda x), x>0, \text { and } \\
\text { Levy symbol } \psi(\theta)=-\gamma \log (1-\theta / \lambda) .
\end{gathered}
$$

The Gamma process has marginal distributions which follow the (continuous) Gamma distribution. Its parameters are gamma $(\gamma)$ which controls the jump intensity, and lambda $(\lambda)$ which controls the inverse jump size. We notate it as $X(t)=\Gamma(t ; \gamma, \lambda)$.

Another process of interest is the Variance Gamma process which is the difference of two Gamma processes

$$
X(t)=V G\left(t ; \gamma, \lambda_{d}, \lambda_{u}\right)=\Gamma\left(t ; \gamma, \lambda_{u}\right)-\Gamma\left(t ; \gamma, \lambda_{d}\right) .
$$

This process was first used in finance by Madan et al (1998), and for modelling credit by Moosbrucker in 2006.

\section{Credit models for single names}

We begin with a simple structural model for a single credit name. We define the value of the firm, or a proxy for it, as the log-Gamma process

$$
S_{t}=S_{0} \exp (-\Gamma(t ; \gamma, \lambda)+\mu t), \text { where } \mu=\gamma \log \left(1+\lambda^{-1}\right) .
$$

Thus we are assuming that $S(t)$ is a positive martingale with up-drift and down-jumps. This simple model assumes that "no news is good news". We also assume that the entity defaults when $S(t)$ goes below a threshold $c$.

We note two extreme cases. As gamma tends to infinity, and $\lambda \sim \sqrt{\gamma} / \sigma$, then $S(t)$ tends to the log-normal process 


$$
S_{t}=S_{0} \exp \left(\sigma W_{t}-\frac{1}{2} \sigma^{2} t\right)
$$

At the other extreme, as gamma tends to zero, and $\lambda \sim \exp (-h / \gamma)$, then $S(t)$ becomes the constant default-intensity model

$$
S_{t}=S_{0} \exp (h t) I(\tau>t), \text { where } \tau \sim \exp (h) \text {, the exponential default time. }
$$

These two cases represent the archetypal extremes of credit defaults. In the log-normal case, spreads widen continuously until default, giving warning of the impending default. (Argentina is a relevant example.) In the second case, spreads never change and defaults happen with no warning at all. (Parmalat was more like this.) The gamma parameter lets us model a situation somewhere in between these extremes.

For calculating the default probability we need to evaluate

$$
p_{T}(c):=P\left(\inf _{t \leq T} S_{t}<c\right)=P\left(\sup _{t \leq T}(\Gamma(t ; \gamma, \lambda)-\mu t)>k:=\log \left(S_{0} / c\right)\right) .
$$

There are two ways of doing this. The simplest is just to use the european approximation:

$$
p_{T}(c) \cong P\left(X_{T}>k\right) \text {, where } X_{t}=\Gamma(t ; \gamma, \lambda)-\mu t .
$$

A more sophisticated approach is to estimate the actual barrier probability using numerical methods. This is more computationally intensive than the european approximation, but can be used in tests to monitor the accuracy level of the european approximation.

The european approximation will not give identical results to the barrier-style method for the same parameters. Instead we would like to be able to calibrate the european-style method so that the european method with european-style parameters closely matches the barrier-style method using barrier-style parameters. If that could be done, then we could have confidence that the european-calibrated european-style prices were a good match for the actual barrier prices of trades.

Example - term structure of a single credit

The graph below shows the 10y term structure of credit default swap spreads under three different modelling assumptions. Parameters have been chosen to match approximately the shape of the CDX 125 S7 index spreads as at 24-Nov-2006. The curves show:

(1) Gamma model, with barrier pricing method, and (barrier-calibrated parameters) of $\gamma$ at $49 \%$, and $\lambda$ at 3.18 .

(2) Gamma model, with european pricing method, and (european-calibrated parameters) of $\gamma$ at $49 \%$, and $\lambda$ at 1.50 .

(3) Brownian motion barrier model, $X(t)=W(t)+\mu t$, defaulting when $X(t)$ hits a barrier, with $\mu$ set to 0.153 .

The basket's index spreads are also shown. We have deliberately constrained the important gamma parameter in the european-calibration to equal the barrier-calibration's gamma, but allowed lambda to calibrate separately. 


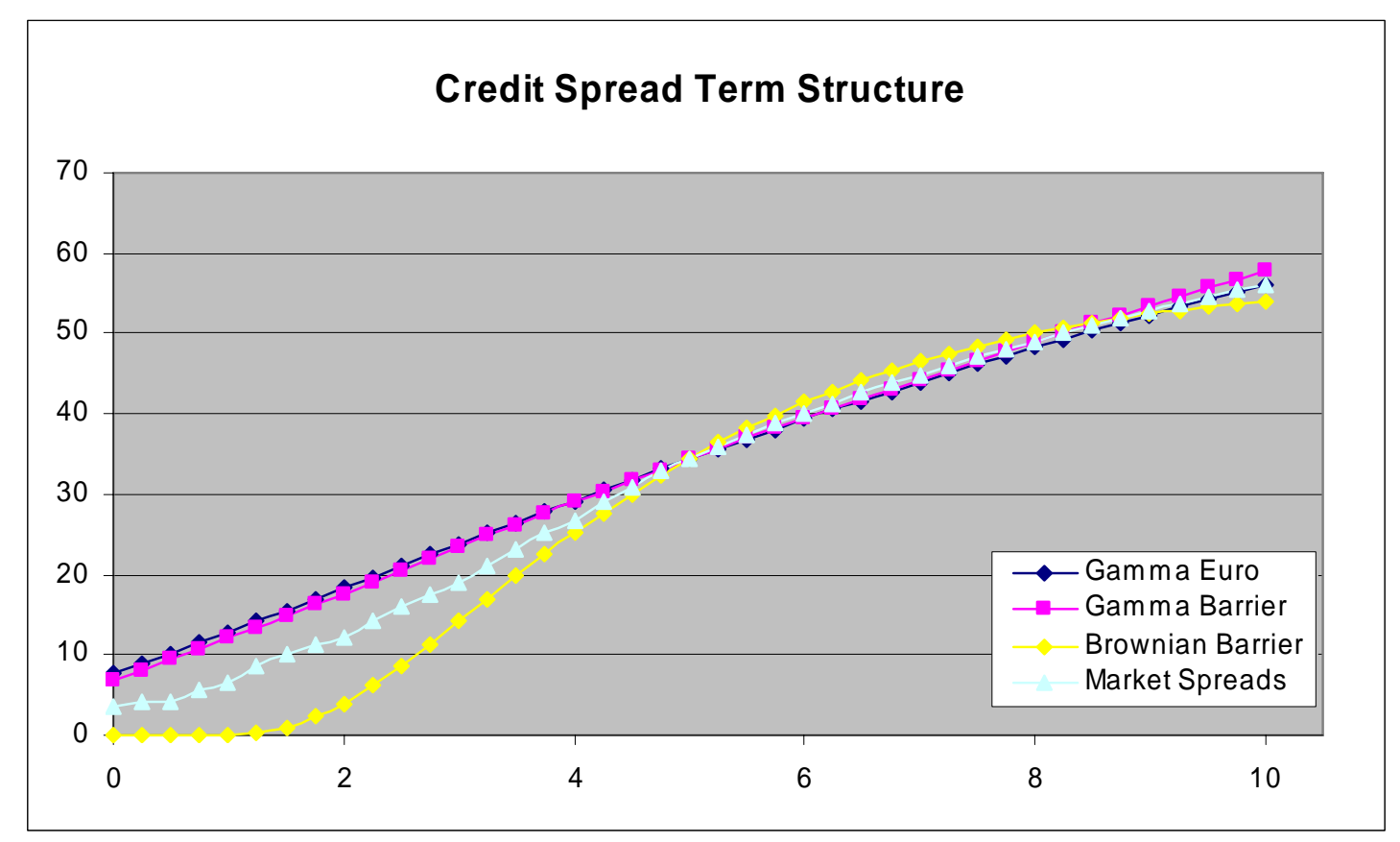

We see that the european and barrier versions of the Gamma model are very similar, and are a rough fit to the actual market curve. The Brownian curve also approximates the market curve, but it underestimates the market spreads at the short end. We do not need the fit to the market curve to be exact, as we will allow the threshold to have a term structure, but it is important that the fit is reasonable to justify the basic form of the model.

For this paper, we will use the european approximation throughout, but it is possible to rework the results with the slower barrier formula. We will also check the barrier formula on CDO prices below.

\section{Extensions}

We can extend the basic model in a number of ways.

(a) We can add "good news" jumps by using the Variance Gamma process instead of the Gamma process.

(b) We can add a global catastrophe term with a low-intensity high-impact global factor (such as a Poisson process or other Gamma process).

(c) We can add a Brownian motion term to get continuous random movement as well.

We can also add various combinations of these, such as Brownian-Variance-Gamma, Catastrophe-Variance-Gamma, and so on. In fact any Levy process can be used in our CDO modelling, as we shall now describe.

\section{Portfolio credit models}

We need to extend our single-name model to a multi-variate correlated model of a portfolio of names. To do this, we need a way of generating multi-variate Levy processes with a given marginal distribution.

\section{Lemma. Multivariate Levy construction}

For any Levy process $X(t)$, any integer $n$, and any non-negative correlation $\phi$, we can construct a set of $n$ Levy processes $X_{1}(t), X_{2}(t), \ldots, X_{n}(t)$, such that each $X_{i}(t)$ has the same distribution as $X(t)$, and the correlation between $X_{i}(t)$ and $X_{j}(t)$ is $\phi$ for all distinct $i$ and $j$.

\section{Proof.}


Start by making $n+1$ independent copies of $X(t)$, called $X_{g}(t), \tilde{X}_{1}(t), \ldots, \tilde{X}_{n}(t)$. Then define

$$
X_{i}(t)=X_{g}(\phi t)+\tilde{X}_{i}((1-\phi) t)
$$

Then, by the stationarity and independent-increment properties of Levy processes, it is immediate that $X_{i}(t)$ is a Levy process with the same distribution as $X(t)$. If $X(t)$ has second moments, then

$$
\begin{gathered}
\operatorname{Var}\left(X_{i}(t)\right)=\sigma^{2} t, \text { for some } \sigma, \text { and hence } \\
\operatorname{Cov}\left(X_{i}(t), X_{j}(t)\right)=\sigma^{2} \phi t, \text { so } \operatorname{Corr}\left(X_{i}(t), X_{j}(t)\right)=\phi .
\end{gathered}
$$

An alternative construction is that we construct the global factor $X_{g}(t)$ as a Levy process with Levy symbol $\psi_{g}$, and $\tilde{X}_{i}(t)$ as IID Levy processes with Levy symbol $\psi_{i}$, where

$$
\psi_{g}(\theta)=\phi \psi(\theta), \psi_{i}(\theta)=(1-\phi) \psi(\theta) \text {, and } X_{i}(t)=X_{g}(t)+\tilde{X}_{i}(t)
$$

Even if $X(t)$ does not have second moments, this representation has the interpretation that a fraction $\phi$ of movements of a single name are due to global effects which affect all other names too.

Given this lemma, we can formulate our CDO model as follows.

We construct a correlated set of state variables from some independent Gamma processes as

$$
X_{i}(t)=-\Gamma_{g}(t ; \phi \gamma, \lambda)-\Gamma_{i}(t ;(1-\phi) \gamma, \lambda)
$$

This decomposes the log-value of the entity into a global and an idiosyncratic Gamma process. We assume, as before, that the entity has defaulted by time $t$, if $X_{i}(t)$ is below a threshold. This threshold can be calibrated to match precisely the survival probability of the entity to that date.

We note that the lambda parameter is redundant, due to scaling. So the Gamma model has only two parameters - gamma and phi.

As an aside, we can reformulate the Gaussian copula as a combination of global and idiosyncratic normal random variables as

$$
X_{i}=\sqrt{\rho} Z_{g}+\sqrt{1-\rho} Z_{i}
$$

This itself can be rewritten as a sum of time-changed Brownian motions as

$$
X_{i}(t)=W_{g}(\rho t)+W_{i}((1-\rho) t)
$$

which matches our general Levy multivariate structure.

\section{Calibration and model comparison}

Our general calibration procedure is take the market's tranche capital structure for a given liquid basket and optimise the model's parameters to achieve the best fit.

The objective function we used is the root mean-squared error, 


$$
V(\gamma, \phi)=\left(\frac{1}{n} \sum_{t r \neq e q}\left(M k t_{t r}-\text { Model }_{t r}\right)^{2}\right)^{1 / 2}
$$

where the sum is taken over the $n$ non-equity tranches of the capital structure, and Mkt(tr) and Model(tr) are respectively the market and model par spreads for tranche tr. This scheme uses a simple weighting, which ignores equity, but other weighting schemes can be used to redistribute the pattern of fitting errors.

The results of the calibration for the CDX 125 S7 basket, as at 28-Nov-2006, are shown in the table.

\begin{tabular}{|c|r|r|r|r|r|r|}
\hline & \multicolumn{2}{|c|}{ 5y CDX S7 } & \multicolumn{2}{|c|}{ 7y CDX S7 } & \multicolumn{2}{c|}{ 10y CDX S7 } \\
\hline Tranche & Market & Model & Market & Model & Market & Model \\
\hline $0 \%-3 \%$ & $24.6 \%$ & $26.6 \%$ & $40.6 \%$ & $44.5 \%$ & $51.1 \%$ & $52.7 \%$ \\
\hline $3 \%-7 \%$ & 91.0 & 90.5 & 210.0 & 210.2 & 426.0 & 427.2 \\
\hline $7 \%-10 \%$ & 18.4 & 19.4 & 46.8 & 46.1 & 110.0 & 109.1 \\
\hline $10 \%-15 \%$ & 6.5 & 7.3 & 19.0 & 20.2 & 51.5 & 54.0 \\
\hline $15 \%-30 \%$ & 3.1 & 1.6 & 6.0 & 5.9 & 14.8 & 21.0 \\
\hline $30 \%-100 \%$ & 1.4 & 0.04 & 2.3 & 0.4 & 3.9 & 2.4 \\
\hline Fit score (bp) & & $\mathbf{1 . 1}$ & & $\mathbf{1 . 1}$ & & $\mathbf{3 . 2}$ \\
\hline
\end{tabular}

The best fit score is also shown (the root-mean-square error in basis points), which excludes the equity error. The general fitting quality is quite good, but not perfect. The larger errors are in

- equity tranches, which are excluded from the fitting objective. Especially in 7y which has a $4 \%$ error. This can be reduced by including the equity tranche in the objective function, at the expense of the other tranches.

- senior and super-senior. The model's ratio of the senior spread to the super-senior spread has values 35,15 and 9 , compared with the market's values of 2,3 and 4 . We have not been able to find any model which comes close to these ratios of market prices. Those market price ratios are close to implying an arbitrage opportunity, as buying senior protection and selling (more) super-senior protection will make money for many plausible scenarios on recovery rates and conditional default probabilities.

The equivalent results for iTraxx 125 S6, also at 28-Nov-2006, are shown here.

\begin{tabular}{|c|r|r|r|r|r|r|}
\hline & \multicolumn{2}{|c|}{ 5y iTraxx S6 } & \multicolumn{2}{|c|}{ 7y iTraxx S6 } & \multicolumn{2}{c|}{ 10y iTraxx S6 } \\
\hline Tranche & Market & Model & Market & Model & Market & Model \\
\hline $0 \%-3 \%$ & $14.0 \%$ & $15.5 \%$ & $28.9 \%$ & $30.9 \%$ & $42.4 \%$ & $46.0 \%$ \\
\hline $3 \%-6 \%$ & 63.0 & 62.6 & 144.0 & 144.0 & 345.5 & 346.9 \\
\hline $6 \%-9 \%$ & 17.3 & 17.7 & 42.5 & 42.5 & 109.0 & 106.8 \\
\hline $9 \%-12 \%$ & 7.0 & 7.9 & 21.3 & 20.8 & 47.8 & 51.1 \\
\hline $12 \%-22 \%$ & 2.9 & 2.7 & 7.3 & 8.1 & 15.0 & 19.6 \\
\hline $22 \%-100 \%$ & 1.2 & 0.1 & 1.9 & 0.6 & 3.4 & 1.3 \\
\hline Fit score (bp) & & $\mathbf{0 . 7}$ & & $\mathbf{0 . 7}$ & & $\mathbf{3 . 0}$ \\
\hline
\end{tabular}

The fitting behaviour is similar to CDX.

The parameter values which achieved these fits were

\begin{tabular}{|l|r|r|r|r|}
\hline & CDX Gamma & CDX Phi & iTraxx Gamma & iTraxx Phi \\
\hline $5 y$ & $152.5 \%$ & $8.9 \%$ & $135.5 \%$ & $9.4 \%$ \\
\hline $7 y$ & $49.2 \%$ & $10.6 \%$ & $60.3 \%$ & $11.5 \%$ \\
\hline $10 y$ & $15.5 \%$ & $19.5 \%$ & $45.8 \%$ & $13.7 \%$ \\
\hline
\end{tabular}


These parameters have the typical behaviour of decreasing gamma with maturity, and increasing phi. Gamma levels are also relatively high in November 2006, which we shall discuss below.

We can also perform the calibration using the barrier formula, rather than the european approximation. In this case the 5y CDX fitted spreads are: $25.9 \%, 90.5,19.4,7.2,1.5$, and 0.04 . These are almost identical to the european fitted values, though with slightly different calibrated parameters (Gamma 158\%, Phi 10\%, Lambda 50\%), so the net effect of the barrier formula is similar to a small change in co-ordinates rather than different model prices.

We have also performed a comparison test of a variety of Levy process models. The models used were: the basic Gamma model (down jumps only); Variance Gamma (asymmetric up and down jumps); Brownian Gamma (down jumps plus diffusion); Brownian Variance Gamma (symmetric up and down jumps plus diffusion); Catastrophe Gamma (down jumps plus global catastrophe factor); Catastrophe Variance Gamma (symmetric up and down jumps plus catastrophe). Additionally the Gaussian copula, without Base Correlation, was included for reference.

All the models were calibrated to historical market data over the period 12 October 2005 to 5 April 2006, using one date per week.

\begin{tabular}{|c|c|c|c|c|c|c|c|}
\hline Model & $\begin{array}{l}\text { CDX } \\
5 y\end{array}$ & $\begin{array}{l}\text { CDX } \\
7 y\end{array}$ & $\begin{array}{l}\text { CDX } \\
10 y\end{array}$ & $\begin{array}{l}\text { iTraxx } \\
5 y\end{array}$ & $\begin{array}{l}\text { iTraxx } \\
7 y\end{array}$ & $\begin{array}{l}\text { iTraxx } \\
10 y\end{array}$ & $\begin{array}{l}\text { Average } \\
\text { (bp) }\end{array}$ \\
\hline Catastrophe Gamma & 1.4 & 7.9 & 15.4 & 1.1 & 7.0 & 8.7 & 6.9 \\
\hline Variance Gamma & 2.9 & 9.6 & 15.7 & 2.9 & 9.6 & 7.0 & 8.0 \\
\hline Gamma & 3.3 & 7.7 & 17.2 & 3.2 & 6.8 & 17.0 & 9.2 \\
\hline Brownian Gamma & 4.7 & 11.1 & 18.3 & 3.9 & 9.2 & 13.8 & 10.2 \\
\hline Brownian Var Gamma & 2.8 & 21.9 & 44.2 & 2.3 & 18.2 & 40.6 & 21.7 \\
\hline Cat Var Gamma & 1.4 & 28.6 & 48.1 & 1.0 & 26.4 & 34.7 & 23.4 \\
\hline Gaussian copula & 38.9 & 66.1 & 76.3 & 33.6 & 75.7 & 83.9 & 62.4 \\
\hline
\end{tabular}

The average pricing error along the capital structure (these runs included the equity tranche) is shown for each basket/maturity combination, and the total average is in the rightmost column.

The Gaussian copula, as expected, performs badly with an average spread error of $62 \mathrm{bp}$. All the Levy based models are significant improvements on the Gaussian copula. The BrownianVariance-Gamma and Catastrophe-Variance-Gamma, which both have symmetric up and down jumps, are relatively poor performers. This suggests that it is important that up-jumps be different to (and smaller than) the down-jumps.

Interestingly, the best four models have quite similar scores, lying in the range 7-10bp. Of these, the Gamma model has the fewest parameters and greater parameter stability. This parsimony encourages us to focus on the Gamma model as our favoured model at this stage.

We can calculate the historical gamma and phi parameters for the Gamma model. We have data which lets us do this daily from 1 April 2005 up to 20 November 2006. The gamma parameter is graphed first, then the phi parameter. 


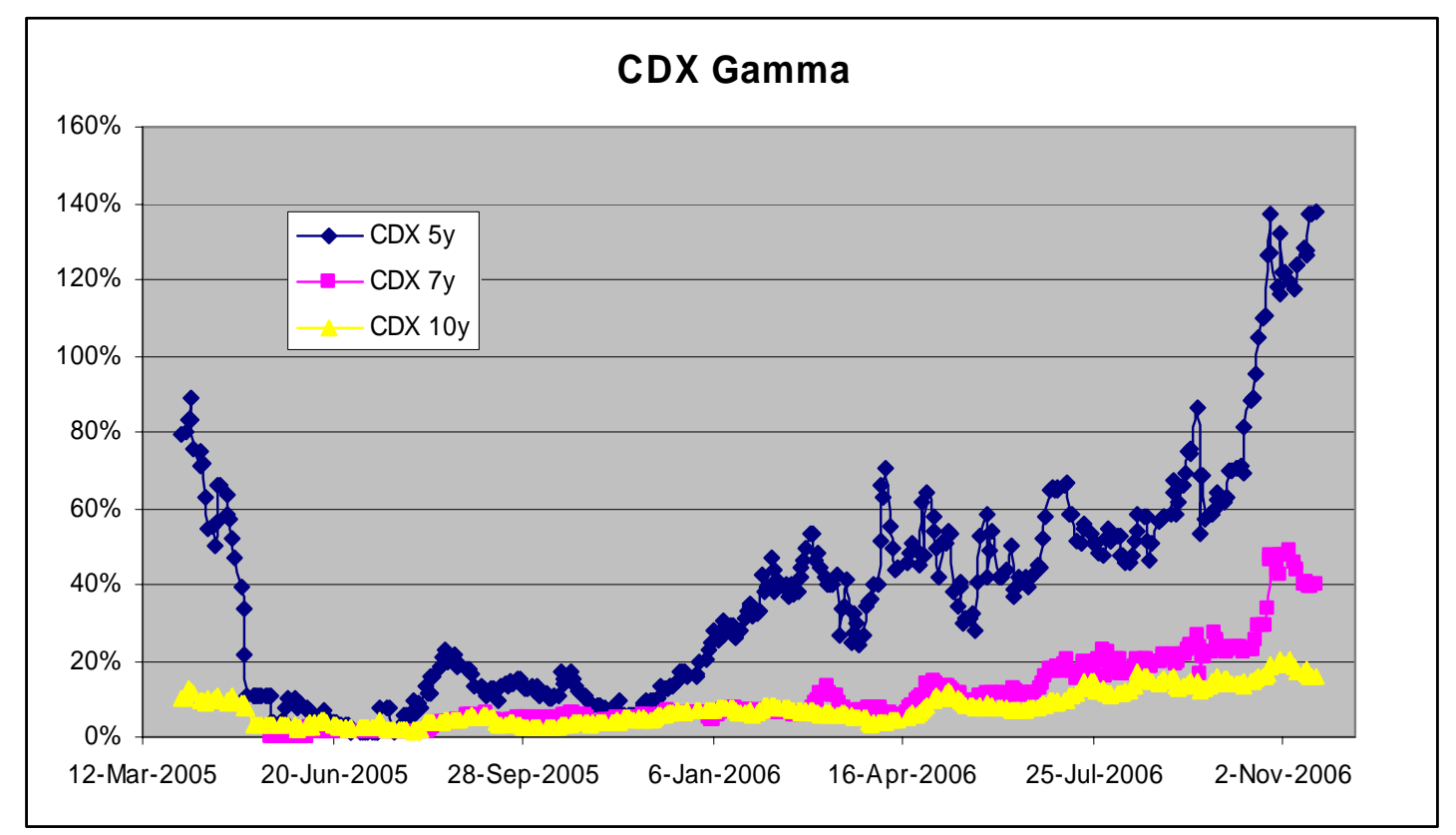

We see that the auto crisis of May 2005 was linked to a crash in the value of gamma from about $80 \%$ down to $10 \%$. Since then, gamma has been gradually increasing, with a faster increase in November 2006, which some market commentary called the "reverse correlation crisis" (Creditflux 3 November 2006).

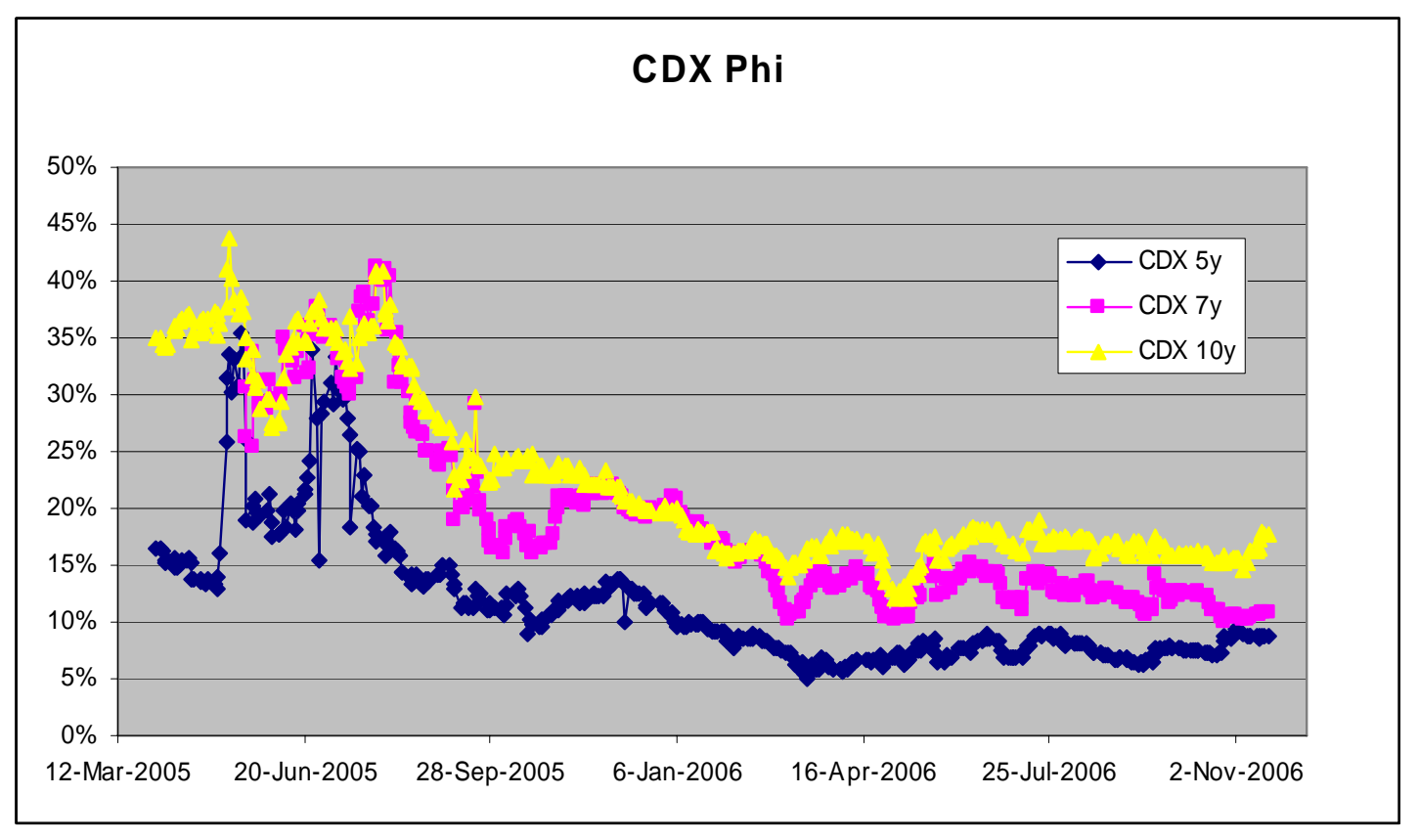

Phi was relatively stable during the auto crisis, though noisier shortly afterwards, and it trended downwards in the second half of 2005. During 2006 phi has been very stable.

\section{Parameter risks and hedging}

Risks are divided into credit spread risks and correlation skew risks. Because the model is "bottom up", it readily shows credit spread risk (and recovery rate risk) to every name in the basket. This compares with "top down" approaches, such as Brigo et al (Nov 2006), which focus on the loss distribution and cannot easily give the credit risk of each name. 
The correlation risk is given by the risks to the parameters. We can see the parameter risks of the CDX 125 S7 basket along its capital structure. The table shows the risk, tranche by tranche, for a $\$ 10$ million buy-protection par $5 y$ trade to a $1 \%$ increase in gamma and phi levels respectively.

\begin{tabular}{|c|r|r|}
\hline & d/dGamma & d/dPhi \\
\hline $0 \%-3 \%$ & -799 & $-50,532$ \\
\hline $3 \%-7 \%$ & 1,384 & 15,270 \\
\hline $7 \%-10 \%$ & 135 & 8,142 \\
\hline $10 \%-15 \%$ & -87 & 4,423 \\
\hline $15 \%-30 \%$ & -110 & 1,612 \\
\hline $30 \%-100 \%$ & -20 & 137 \\
\hline
\end{tabular}

We see that when gamma increases, then the equity and senior spreads decrease and the mezz spreads increase. Increasing gamma moves value from the edges of the capital structure into the middle.

When phi increases, equity spreads decrease and the mezz and senior spreads increase. Increasing phi moves value from the very junior tranches to the more senior.

In base correlation terms, increasing gamma flattens the base correlation curve and increasing phi moves the base correlation curve upwards. A useful interpretation is that phi controls the average level of the base correlation curve, and gamma controls its slope. Indeed, as we saw earlier, an infinite gamma corresponds to the Gaussian copula and a perfectly flat base correlation curve.

For risk management of a CDO portfolio we can hedge gamma and phi (by maturity) to flatten our risk. This is an advance on base correlation, where hedging is based on tranche-bytranche inventory management. Having a model allows parameter-based hedging, which is similar in spirit to the hedging of stochastic vol parameters in the interest-rate skew market. It also allows us to hedge different parts of the capital structure against each other. We can also price bespoke baskets using IG parameters (adapted as necessary), and hedge the risk with IG tranches.

Case study: Auto crisis May 2005

Prior to the auto crisis, many hedge fund investors had a "positive carry" trade, which involved selling equity protection and buying mezz protection. This was about flat in credit spread risk, and long correlation. It was not appreciated at the time, but this was also a long gamma position, as we can see from the risk table above. The crisis was marked by a general spread widening and a strong gamma sell-off (see gamma history plot above). This widened equity spreads (which are short gamma), but tightened mezzanine spreads (which are long gamma). So the investors (who were long gamma) lost money.

Hindsight makes it easy to make the correct decisions in retrospect, but the Gamma model could have provided some risk management information beforehand. It would have warned potential investors both that they were running an exposed position in correlation skew (gamma), and also that gamma was trading at high levels.

\section{Implementation and other products}

The models presented here can be implemented in a similar way to many existing Gaussian copula implementations. In particular, they do not require Monte Carlo simulation, though it is possible to use it.

Let us formulate the Gaussian copula model in a similar way to our Levy process dynamics as

$$
X_{i}(t)=W_{g}(\rho t)+W_{i}((1-\rho) t),
$$


where $W(g)$ and $W(I)$ are global and idiosyncratic Brownian motions. Thus $X_{i}$ is also a Brownian motion. Let us write $F(x ; t)$ for the common distribution function of $X(t), W_{g}(t)$, and $W_{i}(t)$. A sketch of the implementation could run as follows:

(1) For each time $t$, calculate the threshold $\theta_{i}(t)=F^{-1}\left(p_{i}(t) ; t\right)$, where $p_{i}(t)$ is the default probability of entity $i$ by time $t$.

(2) Integrate over the possible values of the global factor $W(g)$, which has distribution function $F(x ; \rho t)$. We can use either simple methods such as Simpson integration, or more sophisticated quadrature techniques. Both methods use an approximation of the form

$$
E\left(\operatorname{payoff}\left(W_{g}\right)\right) \cong \sum_{k=1}^{m} \alpha_{k} E\left(\text { payoff } \mid W_{g}=y_{k}\right)
$$

where $y(k)$ are a discrete set of values of $W(g)$, and $\alpha(k)$ are some weights.

(3) Given that the global factor $(W(g)=y)$, we calculate the conditional default probabilities of each entity

$$
p_{i}(y, t)=P\left(X_{i}(t) \leq \theta_{i}(t) \mid W_{g}=y\right)=F\left(\theta_{i}(t)-y ;(1-\rho) t\right)
$$

(4) With these conditional default probabilities, we calculate the conditional expectation of the payoff. This is helped by the conditional independence of the entities' values given $W(g)$. The expectation can be performed by approximations such as a normal-approximation to the basket loss, or the ingenious bucket algorithm of Hull and White (2004).

To change from the Gaussian copula to our new Levy models, all we have to do is replace the distribution function $F$ used in steps (1), (2), and (3). Step (4) is unaltered. So the problem reduces to calculating the marginal distribution function for the various models we have used.

\section{Calculating the distribution function}

Gamma model. The Gamma model has marginal gamma distributions. Their distribution is already well approximated. See, for example, section 6.2 of Numerical Recipes (Press et al, 1988). Quadrature integration against a gamma random variable is also possible, as implemented in routine gaulag of Numerical Recipes section 4.5. Run-time performance for the Gaussian copula and the Gamma model should be broadly similar.

For calculating the inverse of the distribution function, it is effective to perform interval bisection to bracket the root initially, since the distribution function is monotonic, and then apply some Newton-Raphson iterations to polish it.

Variance Gamma model. There is a time-change representation of the VG process as

$$
V G\left(t ; \gamma, \lambda, \lambda_{u}\right)=W\left(A_{t}\right)+\mu A_{t}
$$

where $W(t)$ is a Brownian motion, $A(t)$ is a gamma $\Gamma\left(t ; \gamma, \frac{1}{2} \lambda \lambda_{u}\right)$ process, and $\mu$ is the drift $\frac{1}{2}\left(\lambda_{d}-\lambda_{u}\right)$. Thus $P\left(V G\left(t ; \gamma, \lambda, \lambda_{u}\right) \leq x\right)=P\left(Z \sqrt{A_{t}}+\mu A_{t} \leq x\right)$. The probability can then be expressed as an integral conditional on the value of $\Gamma=A(t)$ as

$$
P(Z \sqrt{\Gamma}+\mu \Gamma \leq x)=\int_{0}^{\infty} f_{\Gamma}(y) \Phi\left(\frac{x-\mu y}{\sqrt{y}}\right) d y
$$


This integral can be performed efficiently using the gamma quadrature integration mentioned above. Numerical difficulties may occur when the ratio $\lambda_{u} / \lambda$ is extreme (larger than 10 ), so these cases might be excluded.

Other models, such as Brownian Gamma, can be handled in similar ways.

\section{Performing the optimization}

Gamma model. The optimization for the Gamma model is relatively straightforward. There are only two parameters (gamma and phi) and they both have non-trivial and different effects on the tranche spreads. We use an optimiser similar to the Levenberg-Marquardt method, described in section 15.5 of Numerical Recipes. About half-a-dozen iterations are enough to get a good calibration.

Other models. We use the same optimizer, but the situation is more complicated. The function mapping parameters to tranche spreads is significantly non-linear and the presence of semi-redundant parameters increases the difficulty. For such difficult parameters, we try optimizing whilst keeping that parameter fixed, and then we vary the parameter and optimize again. This is slow but effective.

\section{Other Products}

The model can be extended to price bespoke tranches, bespoke baskets and more exotic products. Bespoke tranches and tranchelets are priced immediately in the same way as standard tranches above. Bespoke basket baskets can also be priced, once we know the values of gamma and phi for the bespoke basket. Those, as ever, have to be estimated from the liquid basket parameters. Investment-grade parameters can be estimated as the average of the CDX and iTraxx parameters, and the high-yield basket CDX HY is also liquid giving an estimate of high-yield parameters. A straightforward bespoke pricing scheme is just to take a convex combination of the IG and HY parameter sets, driven by the spread of the bespoke basket. Bespoke pricing is never certain, so room still remains for trading judgement on a bespoke basket's gamma and phi levels. So uncertainty is reduced down to two simple parameters.

More exotic products, such as CDO-squared and long-short CDO, can also be priced under the model. By conditioning on the global factor, the product can be priced using the conditional independence of the names.

\section{Summary and conclusions}

We have presented a family of Levy process models for single-name credits and baskets. Of these, a simple and effective model is the Gamma model. This model has two parameters, which control respectively the average level and slope of the base correlation curve.

The model is tractable to implement, with straightforward calibration. The model can price bespoke baskets and tranchelets, as well as exotic products such as $\mathrm{CDO}^{\wedge} 2$ and long-short CDOs. Risk management is provided with risks given to both every individual credit and the two skew parameters. This allows hedging of skew parameters across tranches and baskets.

Using the european approximation to implement the model does, in theory, remove its fully dynamic character. But the actual barrier implementation, which is more dynamic, produces very similar calibrated prices. 


\section{References}

David Applebaum (2004) "Levy Processes and Stochastic Calculus", Cambridge University Press.

Damiano Brigo, Andrea Pallavicini and Roberto Torresetti, "Calibration of CDO Tranches with the Dynamical Generalized-Poisson Loss Model" (Nov 2006).

Creditflux newsletter (3 Nov 2006), "Rated equity deals put on hold as second correlation crisis bites".

John Hull and Alan White (Sep 2004) "Valuation of a CDO and an $n$th to Default CDS Without Monte Carlo Simulation", Journal of Derivatives 12, 2 (Winter 2004) pp 8-23.

(http://www.rotman.utoronto.ca/ hull/DownloadablePublications/HullWhiteCDOPaper.pdf)

Mark Joshi and Alan Stacey (May 2005) "Intensity Gamma: a new approach to pricing portfolio credit derivatives", preprint. (http://www.quarchome.org/ig.pdf)

Elisa Luciano and Wim Schoutens (Dec 2005) "A Multivariate Jump-Driven Financial Asset Model", preprint. (http://perswww.kuleuven.ac.be/ u0009713/multivg.pdf)

Dilip Madan, Peter Carr and Eric Chang (1998) "The Variance Gamma Process and Option Pricing", European Finance Review, Vol2, No 1 (Sep), pp 79-105.

(http://www.rhsmith.umd.edu/faculty/dmadan/vgoptj98.pdf)

Thomas Moosbrucker (Jan 2006) "Pricing CDOs with Correlated Variance Gamma Distributions", Centre for Financial Research, Univ. of Cologne, colloquium paper (http://www.cfr-cologne.de/downloads/kolloquium/Moosbrucker\%20-\%20041102.pdf)

William Press, Saul Teukolsky, William Vetterling, Brian Flannery (1988) "Numerical Recipes in C", Cambridge University Press.

Wim Schoutens (Jan 2006), "Jumps in Credit Risk Modelling", presentation at King's College London 31 Jan 2006.

Matthias Winkel (2004), "Introduction to Levy processes", graduate lecture at the Department of Statistics, Univ. of Oxford 22 Jan 2004. (http://www.stats.ox.ac.uk/ winkel//p1.pdf) 


\section{Disclaimer}

\section{Credit Derivatives}

This information has been issued by the Sales/Trading departments of Nomura International plc ("NIplc"), Nomura Securities International, Inc. ("NSI"), and/or its affiliates (collectively, "Nomura"), in order to promote investment services and is provided without compensation. This is not objective investment research as defined by the UK Financial Services Authority ("FSA"), nor is it research under the rules of the U.S. Self Regulatory Organizations of which Nomura is a member. Information contained herein is provided for informational purposes only, is intended solely for your use and may not be quoted, circulated or otherwise referred to without our express consent. This material contains indicative terms only, and should not be considered as an offer to buy or sell securities or other products discussed herein. Any prices, yields and opinions expressed are subject to change without notice. The information is based on sources we believe to be reliable, but we do not represent that it is accurate or complete. We are not your designated investment adviser and this information is therefore provided on the basis that you have such knowledge and experience to evaluate its merits and risks, and are capable of undertaking your own objective analysis of the investment and its suitability to meet your requirements. Nomura and/or connected persons do not accept any liability whatsoever for any direct, incorrect or inconsequential loss arising from any use of the information or its content. Nomura also may have acted as an underwriter of such securities or other products discussed in this material, and may currently be providing investment banking services to the issuers of such securities or products. Nomura and/or its officers, directors and employees, including persons, without limitation, involved in the preparation or issuance of this material may, from time to time, have long or short positions in, and buy or sell, the securities, or derivatives (including options) thereof, of companies mentioned herein, or related securities or derivatives. This material has been approved for distribution in the United Kingdom and European Union by NIplc, which is authorised and regulated by FSA and is a member of the London Stock Exchange. It is not intended for private customers. It is intended only for investors who are "market counterparties" or "intermediate customers" as defined by the FSA, and may not, therefore, be redistributed to other classes of investors. NSI accepts responsibility for the contents of this material when distributed in the United States. Nomura manages conflicts identified through the following: their Chinese Wall, confidentiality and independence policies, maintenance of a Stop List and a Watch List, personal account dealing rules, policies and procedures for managing conflicts of interest arising from the allocation and pricing of securities and impartial investment research and disclosure to clients via client documentation. Further disclosure information is available at http://www.nomura.com/research/. Additional information is available upon request. 